\title{
Involvement of inhibitor kappa B kinase 2 (IKK2) in the regulation of vascular tone
}

\author{
Youngin Kwon ${ }^{1} \cdot$ Soo-Kyoung Choi $^{1} \cdot$ Seonhee Byeon ${ }^{1} \cdot$ Young-Ho Lee ${ }^{1}$
}

Received: 6 November 2017 / Revised: 22 March 2018 / Accepted: 23 March 2018 / Published online: 21 May 2018

(c) United States \& Canadian Academy of Pathology 2018

\begin{abstract}
Inhibitor kappa B kinase 2 (IKK2) plays an essential role in the activation of nuclear factor kappa B (NF-kB). Recently, it has been suggested that IKK2 acts as a myosin light chain kinase (MLCK) and contributes to vasoconstriction in mouse aorta. However, the underlying mechanisms are still unknown. Therefore, we investigated whether IKK2 acts as a MLCK or regulates the activity of myosin light chain phosphatase (MLCP). Pressure myograph was used to measure vascular tone in rat mesenteric arteries. Immunofluorescence staining was performed to identify phosphorylation levels of MLC (ser19), MYPT1 (thr853 and thr696) and CPI-17 (thr38). SC-514 (IKK2 inhibitor, 50 $\mu \mathrm{M}$ ) induced relaxation in the mesenteric arteries pre-contracted with $70 \mathrm{mM}$ high $\mathrm{K}^{+}$solution or U-46619 (thromboxane analog, $5 \mu \mathrm{M}$ ). The relaxation induced by SC-514 was increased in the arteries pre-contracted with U-46619 compared to arteries pre-contracted with $70 \mathrm{mM}$ high $\mathrm{K}^{+}$solution. U-46619-induced contraction was decreased by treatment of SC-514 in the presence of MLCK inhibitor, ML-7 (10 $\mu$ M). In the absence of intracellular $\mathrm{Ca}^{2+}$, U-46619 still induced contraction, which was decreased by treatment of SC-514. Furthermore, phosphorylation levels of MLC (ser19) and MYPT1 (thr853) were decreased by treatment of SC-514. IKK2 is involved in the vascular contraction through regulation of MLCP activity by phosphorylating MYPT1 at thr853 in rat mesenteric arteries. These findings suggest IKK2 could be a new pharmacological target for specific therapies of various vascular diseases.
\end{abstract}

\section{Introduction}

Blood circulation plays an important role in supplying oxygen and nutrients to tissues [1]. Microvessels contribute to the maintenance and regulation of blood flow and peripheral resistance [2]. Resistance arteries, which have small diameter $(<400 \mu \mathrm{M}$ in lumen diameter), contribute the generation of the peripheral resistance and thus regulate blood flow [3].

Contractile status of smooth muscle is determined by phosphorylation of $20 \mathrm{kDa}$ myosin regulatory light chain (MLC) $[4,5]$. The MLC phosphorylation is dependent on

These authors contributed equally: Youngin Kwon, Soo-Kyoung Choi.

Young-Ho Lee

yhlee@yuhs.ac

1 Department of Physiology, College of Medicine, Brain Korea 21 Plus Project for Medical Sciences, Yonsei University, Seoul, Korea the balance between myosin light chain kinase (MLCK) and myosin light chain phosphatase (MLCP) activity. The activity of MLCK is dependent on cytosolic $\mathrm{Ca}^{2+}$ levels. The MLCP dephosphorylates MLC, resulting in smooth muscle relaxation. Therefore, without change of cytosolic $\mathrm{Ca}^{2+}$ levels, inhibition of MLCP causes smooth muscle contraction which is called $\mathrm{Ca}^{2+}$ sensitization $[6,7]$. It has been reported that several resistance arteries such as mesenteric, coronary, and cerebral arteries exhibit augmented $\mathrm{Ca}^{2+}$ sensitization [8-11].

The MLCP is an enzyme composed of three subunits, 110-130 kDa myosin-binding subunit (non-catalytic myosin phosphatase target subunit, MYPT1), $38 \mathrm{kDa}$ catalytic subunit (protein phosphatase 1, pp1c $\delta$ ), and $20 \mathrm{kDa}$ unknown subunit $\left(\mathrm{M}_{20}\right)[12,13]$. The MYPT1 has crucial role in targeting myosin filament. The enzyme activity of MLCP is regulated by MYPT1 phosphorylation at thr696 or thr853. There are two major pathways of $\mathrm{Ca}^{2+}$ sensitization mechanisms in smooth muscle. One is RhoA and Rho kinase signaling through $\mathrm{G}_{12 / 13}$ heteromeric $\mathrm{G}$ proteins. Activated Rho kinase directly inhibits MLCP [14]. The other mechanism is phosphorylation of CPI-17 (at thr38), which is 
a potent inhibitor of MLCP. It inhibits MLCP when phosphorylated by Rho kinase or protein kinase C [15].

Inhibitor kappa B kinase 2 (IKK2) plays an essential role in the activation of nuclear factor kappa $\mathrm{B}(\mathrm{NF}-\kappa \mathrm{B})$ [16]. The IKK2 phosphorylates IKB $\alpha$ (inhibitor of kappa $B$ ) protein, which is an inhibitory protein of NF- $\mathrm{KB}$ signaling. Phosphorylated $\mathrm{I} \kappa \mathrm{B} \alpha$ is degraded by the ubiquitinproteasome pathway. As the result, free NF- $\kappa \mathrm{B} /$ Rel family activates transcription of various genes, which participate in the immune and inflammatory response, cell adhesion, growth control, and protection against apoptosis [17-19]. The IKK2 is well known to mediate the innate and adaptive immune response in various cells such as monocyte-derived antigen-presenting cells, bone-marrow-derived dendritic cells, natural killer-like T (NKT) cells, and T lymphocytes [20-23]. In addition, IKK2 is known to be involved in inflammatory responses in human airway smooth muscle $[24,25]$. Recently, it has been suggested that IKK2 acts as another MLCK and contributes to vasoconstriction in rat and mouse aorta [26, 27]. However, the underlying mechanisms are not still well understood. Thus, the purpose of this study is to investigate whether IKK2 acts as another MLCK or regulates the activity of MLCP.

\section{Materials and methods}

All experiments were performed according to the Guide for the Care and Use of Laboratory Animals published by US National Institutes of Health (NIH publication no. 85-23, 2011) and were approved by the Ethics Committee and the Institutional Animal Care and Use Committee of Yonsei University, College of Medicine.

\section{Animal models and tissue preparation}

In this experiments, we used 12-week-old male Sprague Dawley rats. After killed, the mesenteric arteries were dissected and immediately placed in chilled Krebs-Henseleit (KH) solution (composed of (in $\mathrm{mmol} / \mathrm{L}$ ) $\mathrm{NaCl}, 119 ; \mathrm{KCl}$, 4.6; $\mathrm{CaCl}_{2}, 2.5 ; \mathrm{MgSO}_{4}, 1.2 ; \mathrm{KH}_{2} \mathrm{PO}_{4}, 1.2 ; \mathrm{NaHCO}_{3}, 25$; glucose, 11.1). A $95 \% \mathrm{O}_{2}+5 \% \mathrm{CO}_{2}$ gas aerated the $\mathrm{KH}$ solution continuously. Adipose and connective tissues were removed under an optical microscope (model SZ-40, Olympus, Japan). Third-order mesenteric arteries were cut $\sim 3-4 \mathrm{~mm}$ long.

\section{Measurement of the vessel diameter}

A mesenteric artery segment $(200-275 \mu \mathrm{M}$ in inner diameter) was cannulated on a glass micropipette in a pressure myograph (model PS-200-P, Living Systems Instrumentation, Burlington, VT, USA). The heated KH solution was superfused continuously to keep $37^{\circ} \mathrm{C}$. During equilibration period (for $30 \mathrm{~min}$ ), the pressure was maintained at $40 \mathrm{mmHg}$ using pressure-servo control perfusion systems. The lumen diameter was measured using the Soft Edge Acquisition Subsystem (IonOptix, Milton, MA, USA). To contract mesenteric arteries, we used $1 \mu \mathrm{M}$ phenylephrine (PE; Sigma-Adrich, St. Louis, MO, USA), 5 $\mu \mathrm{M}$ U-46619 (Cayman chemicals, Ann Arbor, MI, USA), and high $\mathrm{K}^{+}$solution $(70 \mathrm{mM} \mathrm{KCl}$ in $\mathrm{KH}$ solution). Inhibitors of IKK2, 4-amino-[2,3'-bithiophene]-5-carboxamide (SC-514) and [5-(p-Fluorophenyl)-2-ureido]thiophene-3-carboxamide (TPCA-1) were purchased from Selleckchem, (Houston, TX, USA) and Apexbio (Houston, TX, USA), respectively. A MLCK inhititor, hexahydro-1[(5-iodo-1-naphthalenyl)sulfonyl]-1H-1,4-diazepine hydrochloride (ML-7) (Tocris Bioscience, bristol, UK), was used to exclude possible involvement of classical MLCK. $\mathrm{Ca}^{2+}$-free $\mathrm{KH}$ solution consists of no $\mathrm{Ca}^{2+}, 1 \mathrm{mM}$ ethylene glycol-bis(2-aminoethylether)- $N, N, N^{\prime}, N^{\prime}$-tetraacetic acid (EGTA; Sigma-Adrich) and $5 \mu \mathrm{M}$ cyclopiazonic acid (CPA; Cayman chemicals) to exclude intra and extracellular $\mathrm{Ca}^{2+}$.

\section{Immunofluorescence}

The expression and phosphorylation of proteins in mesenteric arteries were measured by immunofluorescence staining as previously described [28]. The vessels were immediately frozen when contractile and relaxing response became stable at resting state, resting state treated with SC514 , treatment of U-46619, and co-treatment of U-46619 and SC-514. The frozen sections $(5 \mu \mathrm{M})$ were serially collected. Sections were blocked by PBS containing $3 \%$ bovine serum albumin. The specific primary antibodies used are as follows: p-MLC (1:50; catalog no. 3671, Cell Signaling Technology, Danvers, MA, USA), total MLC (1:100; cata$\log$ no. AM09080PU-N, Acris, Herford, Germany), p-CPI17 (1:50; catalog no. SC-17560P, Santa Cruz, Dallas, TX, USA), smooth muscle actin (1:100; catalog no. ab7817, Abcam, Cambridge, MA, USA), p-MYPT1 thr853 (1:100; catalog no. bs-328812, Bioss, Woburn, MA, USA), p-MYPT1 thr696 (1:100; catalog no. bs-3287R, Bioss) and total-MYPT1 (1:100; catalog no. 612164, BD Transduction Laboratories, San Jose, CA, USA). The secondary antibodies used streptavidin conjugated-Alexa Fluor 488 and Alexa Fluor 594 (1:100; catalog nos. A21202 and A21207, Invitrogen, Carlsbad, CA, USA). DAPI was used for nuclear staining (Vector Laboratories, Burlingame, CA, USA). Immunofluorescence images were obtained using an Olympus BX51 microscope (model BX51TF, Olympus, Japan). Immunofluorescence intensity was quantified with Image $\mathbf{J}$ (National Institutes of Health, Bethesda, MD, USA). 


\section{Western blot}

The isolated mesenteric arteries at resting state or resting state with SC-514 or after the treatments of U-46619 with or without SC-514 were frozen in liquid nitrogen and then homogenized in ice-cold lysis buffer, as previously described [29]. Western blot analysis was performed for phosphorylated MLC (p-MLC; 1:500, catalog no. 3671s, Cell Signaling, Boston, MA, USA), total MLC (t-MLC; 1:1000 dilution; catalog no. 3672s, Cell Signaling), and $\beta$-actin (1:2000 dilution; catalog no. ab8226, Abcam). Each lane were loaded with $150 \mu \mathrm{g}$ of protein sample. Western blot results were quantified by densitometric measurement using Image $\mathrm{J}$ (National Institutes of Health).

\section{Data and statistical analysis}

Results are expressed as mean \pm SEM. Comparisons between groups were performed with $t$-tests. Values of $P<0.05$ were considered significant. The concentration-response curve was fitted to sigmoidal curve with a variable slope using four parameters logistic equation in GraphPad Prism (Version 7, GraphPad software, La Jolla, CA, USA). For all experiments measuring diameter, the $n$-values mean number of vessels derived from each different animals. Accordingly, $n$-values also mean number of animals.

\section{Materials}

The general laboratory reagents were purchased from Sigma-Aldrich unless otherwise stated. The SC-514, TPCA-1, ML-7, and CPA were prepared as stock solutions in DMSO (dimethyl sulfoxide, Sigma-Aldrich). The PE was dissolved in distilled water as a stock solution. U-46619 was dissolved in methyl acetate. All the stock solutions were aliquoted and stored at $-20^{\circ} \mathrm{C}$ until use.

\section{Results}

\section{Effect of SC-514 in mesenteric arteries pre-contracted with $\mathrm{PE}$}

We used SC-514, an IKK2 inhibitor, to identify the involvement of IKK2 in vasoconstriction induced by $\mathrm{PE}$ ( $\alpha 1$-adrenergic receptor agonist, $1 \mu \mathrm{M}$ ). The SC-514 was treated in dose-dependent manner in the mesenteric arteries
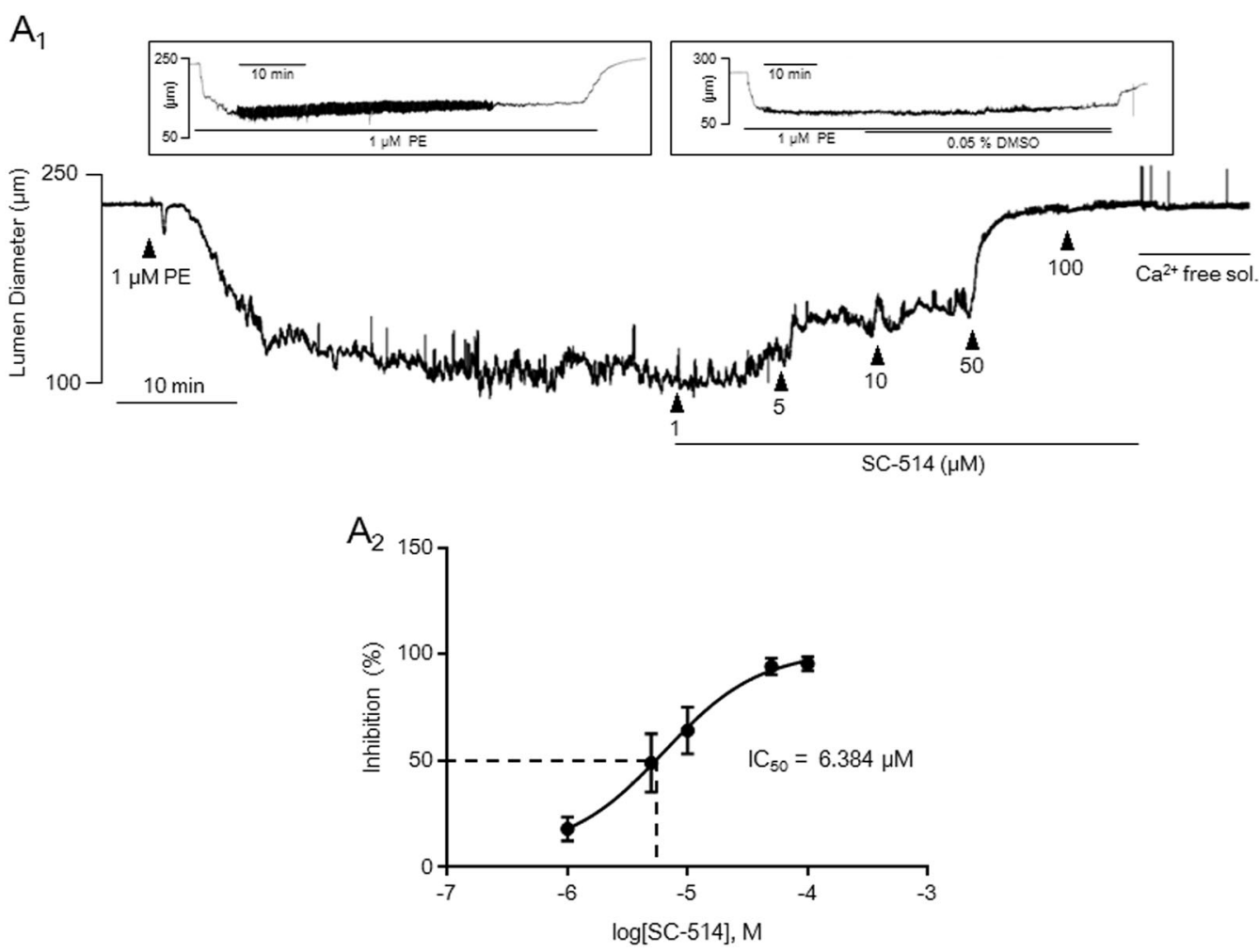

Fig. 1 Dose-response curve for SC-514 in the mesenteric arteries pretreated with PE. $\mathbf{a}_{1}$ Representative recording of relaxing responses to SC-514 in mesenteric arteries. The insets display PE-control trace and
DMSO-control trace. $\mathbf{a}_{2}$ Cumulative dose-response curve for SC-514 in the mesenteric arteries pre-treated with $1 \mu \mathrm{M} \mathrm{PE}(n=5)$ 
A
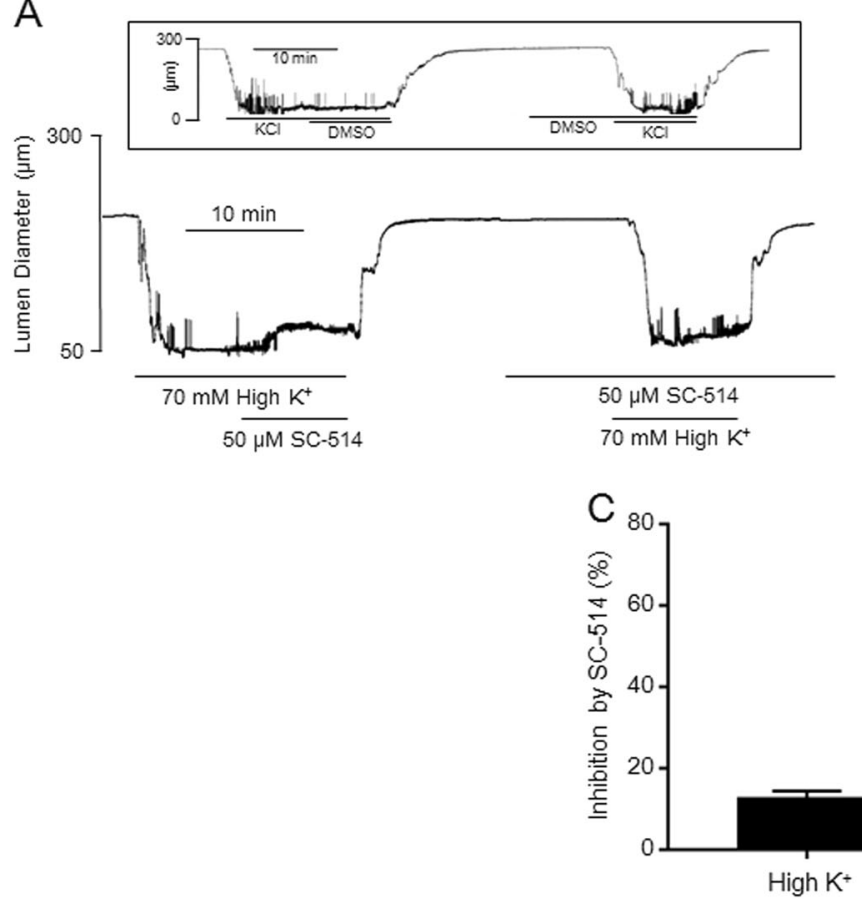

B
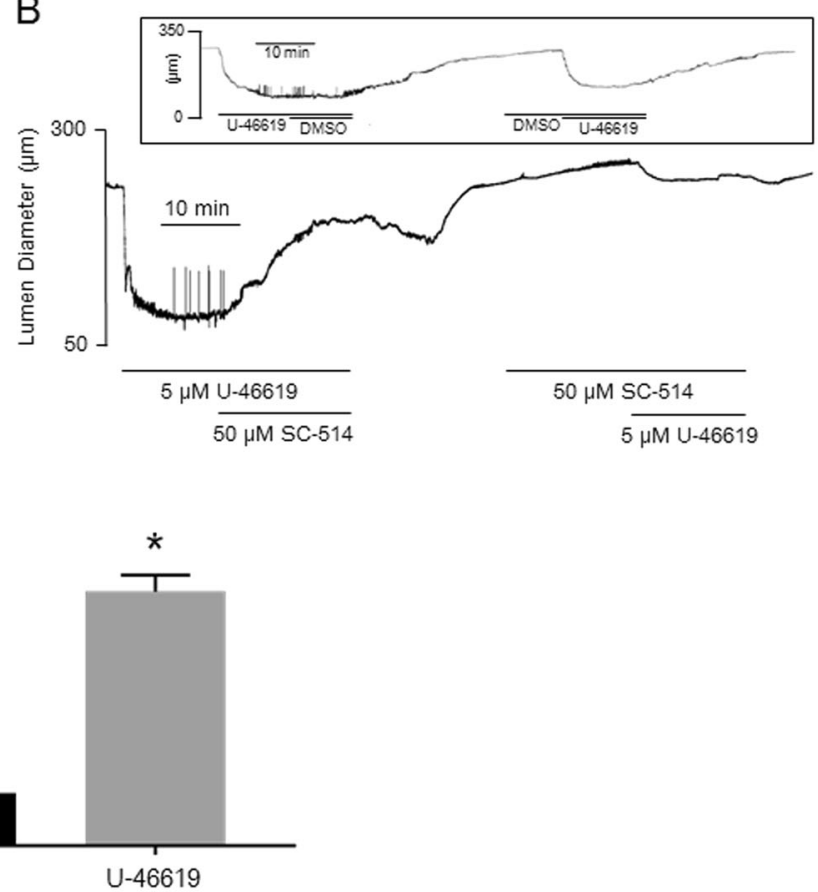

Fig. 2 Effect of SC-514 on high $\mathrm{K}^{+}$- or U-46619-induced contraction in the mesenteric arteries. Representative recordings of vascular responses induced by pre- or post-treatment of $50 \mu \mathrm{M} \mathrm{SC-514}$ in 70 $\mathrm{mM} \mathrm{KCl}$-induced contraction (a) and $5 \mu \mathrm{M}$ U-46619-induced contraction (b). The insets of (a and b) display DMSO-control trace. $\mathbf{c}$ Mean data for the inhibition by SC-514 in high $\mathrm{K}^{+}$- and U-46619induced contraction ( $n=4-5$ for each experiments). $* p<0.05$ for $\mathrm{U}-46619$-induced contraction vs high $\mathrm{K}^{+}$-induced contraction

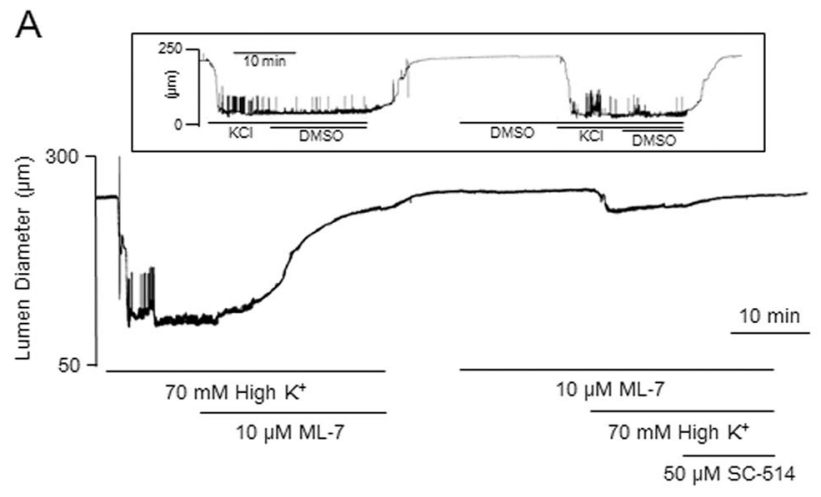

C

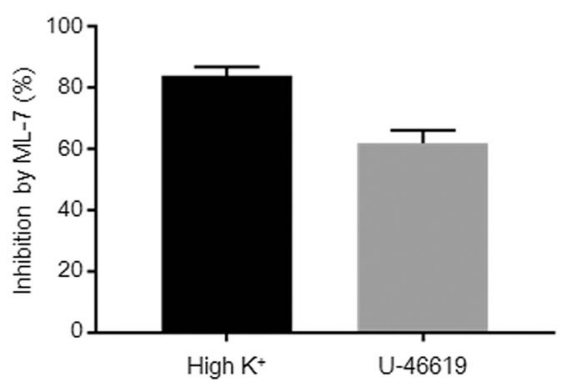

Fig. 3 Effect of SC-514 on high $\mathrm{K}^{+}$- or U-46619-induced contraction in the presence of ML-7. Representative recordings of vascular responses induced by treatment of $50 \mu \mathrm{M} \mathrm{SC}-514$ in $70 \mathrm{mM} \mathrm{KCl}$ induced contraction (a) and $5 \mu \mathrm{M}$ U-46619-induced contraction (b) in the presence of $10 \mu \mathrm{M}$ ML-7. c Percent inhibition of high $\mathrm{K}^{+}$- and
B

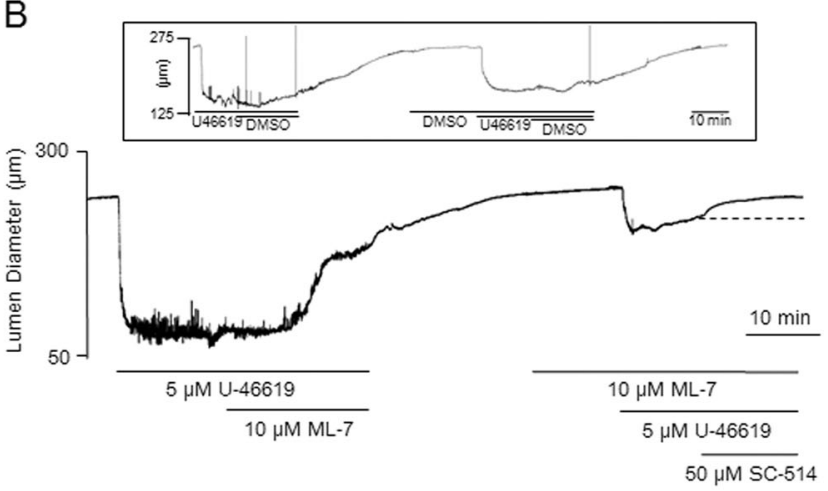

D

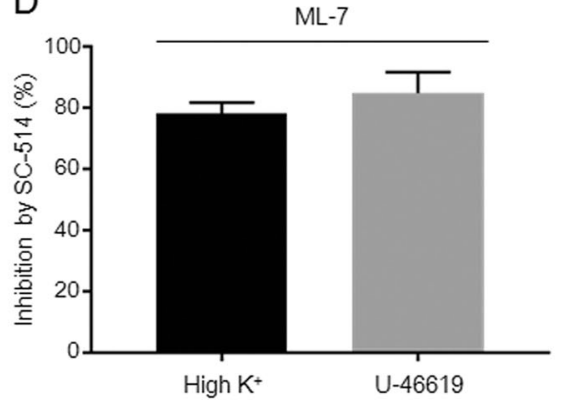

U-46619-induced contraction by ML-7 in mesenteric arteries $(n=5-6$ for each experiments). $\mathbf{d}$ In the presence of ML-7, percent inhibition of high $\mathrm{K}^{+}$- and U-46619-induced contraction by SC-514 in mesenteric arteries ( $n=5-6$ for each experiments) 
Fig. 4 Effect of SC-514 on U-46619-induced contraction in $0-\mathrm{Ca}^{2+}$ condition. $\mathbf{a}_{1}$ Representative recording of relaxing effect of SC-514 on U-46619-induced contraction in normal and $0-\mathrm{Ca}^{2+} \mathrm{KH}$ condition. $\mathbf{a}_{2}$ Percent relative contraction induced by U-46619 in mesenteric arteries $(n=5)$. $* p<0.05$ for $\mathrm{U}-46619$-induced contraction vs U-46619-induced contraction with SC-514 treatment, ${ }^{*} p<0.05$ for $\mathrm{U}$ 46619-induced contraction in $0-\mathrm{Ca}^{2+}$ condition vs U-46619induced contraction with $\mathrm{SC}-514$ treatment in $0-\mathrm{Ca}^{2+}$ condition
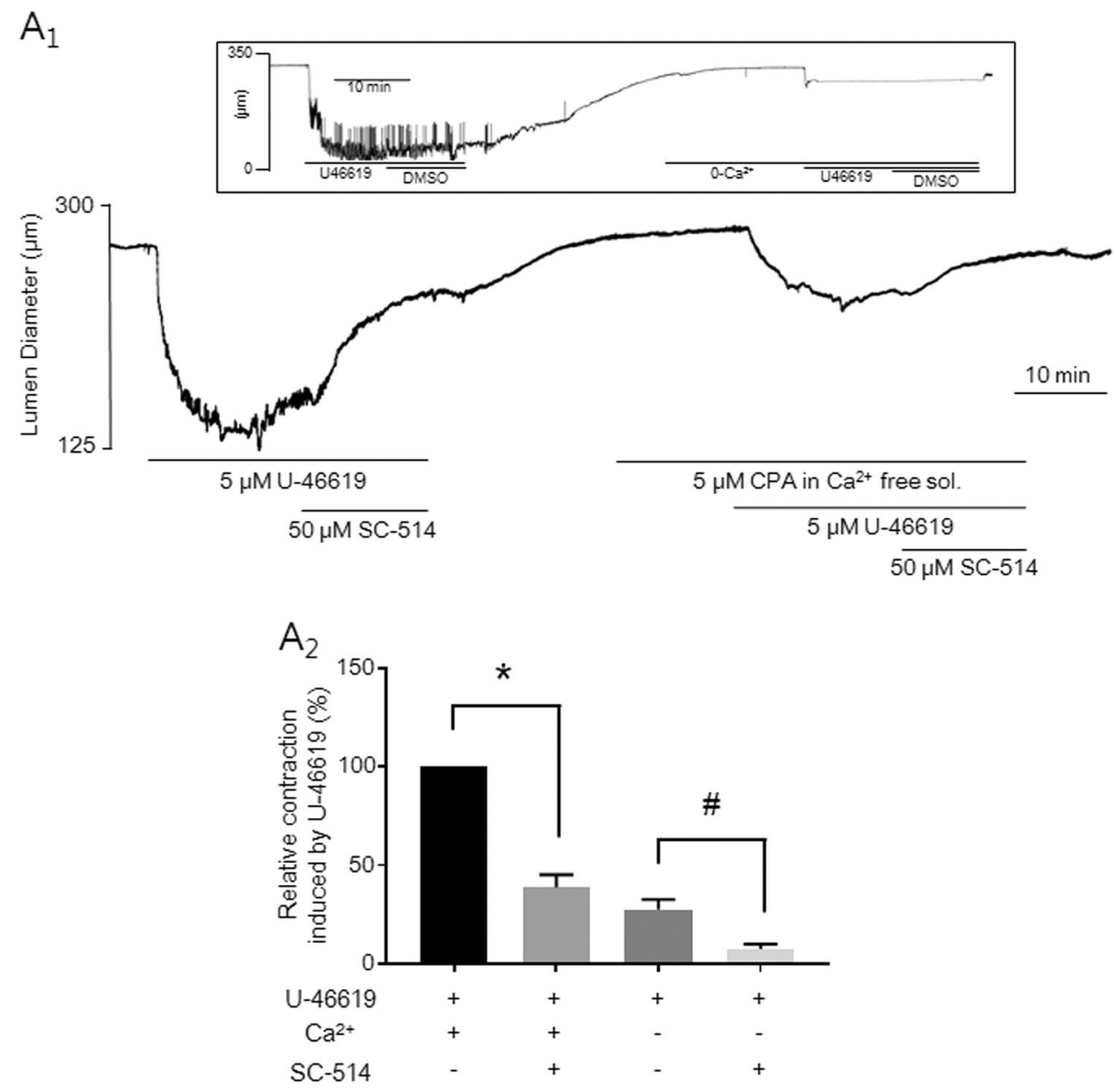

pre-contracted with PE. The SC-514 dose-dependently increased the diameter of mesenteric arteries (Fig. 1). Based on dose-response curve, the dose $(50 \mu \mathrm{M})$ that causes maximal response $(95.3 \pm 1.8 \%)$ was used for the following experiments.

\section{Involvement of IKK2 on the contractile responses in mesenteric arteries}

To inspect involvement of IKK2 on the vasoconstriction, we examined the effect of SC-514 on high $\mathrm{K}^{+}$- and U-46619-induced contraction. Figure 2 shows that SC-514 caused relaxation in mesenteric arteries pre-contracted with $70 \mathrm{mM}$ high $\mathrm{K}^{+}$or U-46619 (a thromboxane A2 receptor agonist, $5 \mu \mathrm{M})$. Interestingly, U-46619-induced contraction was significantly decreased by $\mathrm{SC}-514$ compared to high $\mathrm{K}^{+}$-induced contraction (Fig. 2c). In accordance with these data, when pre-treated with SC-514, U-46619-induced contraction was blocked by $\mathrm{SC}-514$. On the other hand, high $\mathrm{K}^{+}$-induced contraction was not significantly inhibited by pretreatment of SC-514.

\section{Involvement of IKK2 on the contraction, which is not regulated by classic MLCK}

To exclude the relevance of classic MLCK, we used a MLCK inhibitor, ML-7 (Fig. 3). Both U-46619- and high K ${ }^{+}$-induced contraction were inhibited by $10 \mu \mathrm{M}$ ML-7. However, high $\mathrm{K}^{+}$-induced contraction was more reduced by ML-7 compared with U-46619-induced contraction (Fig. 3c). When pre-treated with ML-7, ML-7 blocked both of high $\mathrm{K}^{+}$- and U-46619-induced contraction. The amount of blockage by ML-7 was higher in the high $\mathrm{K}^{+}$-induced contraction than U-46619-induced contraction. In the presence of ML-7, both of these high $\mathrm{K}^{+}$- and U-46619induced contraction were significantly inhibited by SC-514 (Fig. 3d).

\section{Involvement of IKK2 on the $\mathrm{Ca}^{2+}$-independent contraction}

To completely block the activation of classic MLCK, we tested the effect of SC-514 on U-46619-induced contraction in $0-\mathrm{Ca}^{2+} \mathrm{KH}$ solution. Vessels were incubated with $0-\mathrm{Ca}^{2+}$ 

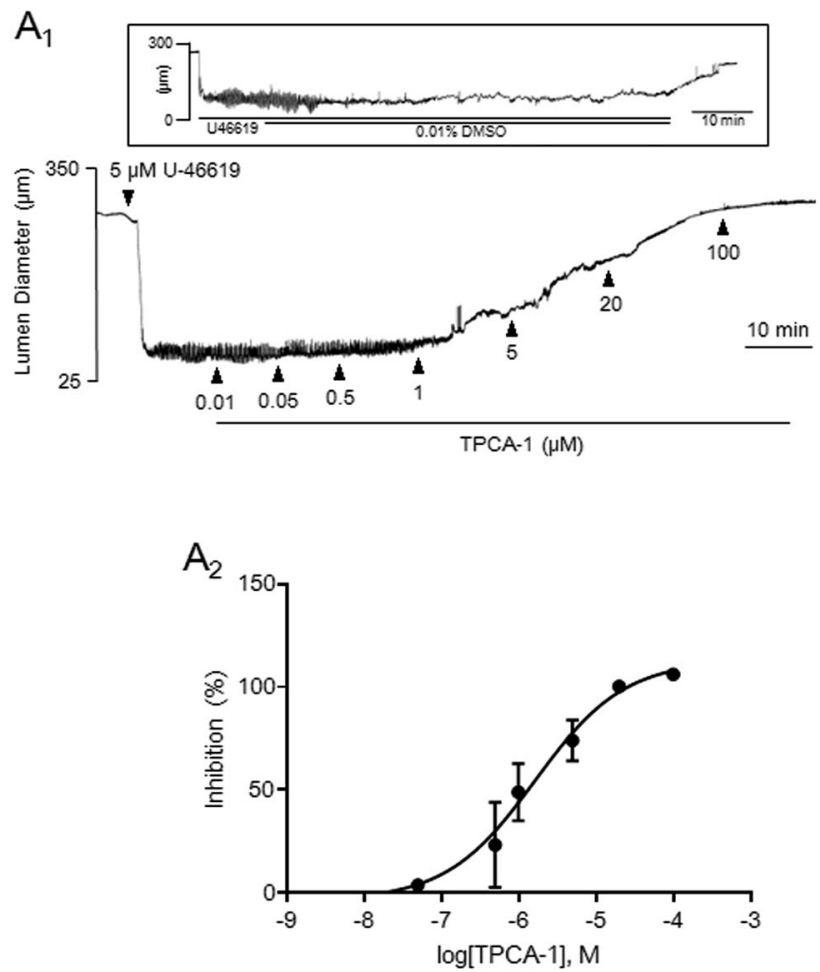

Fig. 5 Dose-response curve for TPCA-1 in the mesenteric arteries pretreated with PE ( $\mathbf{a}_{1}$ and $\mathbf{a}_{2}$ ). $\mathbf{a}_{1}$ Representative recording of relaxing responses to TPCA-1 in mesenteric arteries. $\mathbf{a}_{2}$ Cumulative dose-response curve for TPCA-1 in the mesenteric arteries pre-treated with $1 \mu \mathrm{M}$ PE $(n=4)$. Effect of TPCA-1 on U-46619-induced contraction in $0-\mathrm{Ca}^{2+}$ condition $\left(\mathbf{b}_{1}\right.$ and $\left.\mathbf{b}_{2}\right)$. $\mathbf{b}_{1}$ Representative recording of relaxing effect of TPCA-1 on U-46619-induced contraction in

$\mathrm{KH}$ solution containing $1 \mathrm{mM}$ EGTA and $5 \mu \mathrm{M}$ CPA to eliminate intra and extracellular $\mathrm{Ca}^{2+}$. Under these experimental conditions, U-46619 still induced contraction, which was dilated in response to SC-514 (Fig. 4). To confirm the implication of IKK2, another inhibitor of IKK2 (TPCA-1) was used (Fig. 5). The TPCA-1 was treated in dosedependent manner in the mesenteric arteries pre-contracted with PE. The TPCA-1 dose-dependently increased the diameter of mesenteric arteries (Fig. 5a $\mathrm{a}_{1}$ ). Based on dose-response curve, the concentration $(20 \mu \mathrm{M})$ caused maximal response $(100.3 \pm 0.7 \%)$, which was decided to be used for the following experiments (Fig. 5a $\mathrm{a}_{2}$ ). Under $0-\mathrm{Ca}^{2+}$ conditions, U-46619 still induced contraction, which was dilated in response to TPCA-1 (Fig. $5 b_{1}, b_{2}$ ).

\section{The effect of SC-514 on phosphorylation of MLC, CPI-17, and MYPT1 in the mesenteric arteries}

To investigate whether SC-514 affects phosphorylation levels of MLC, MYPT1, and CPI-17, we performed immunofluorescence staining. We found that phosphorylated MLC (p-MLC, ser19) and phosphorylated MYPT1
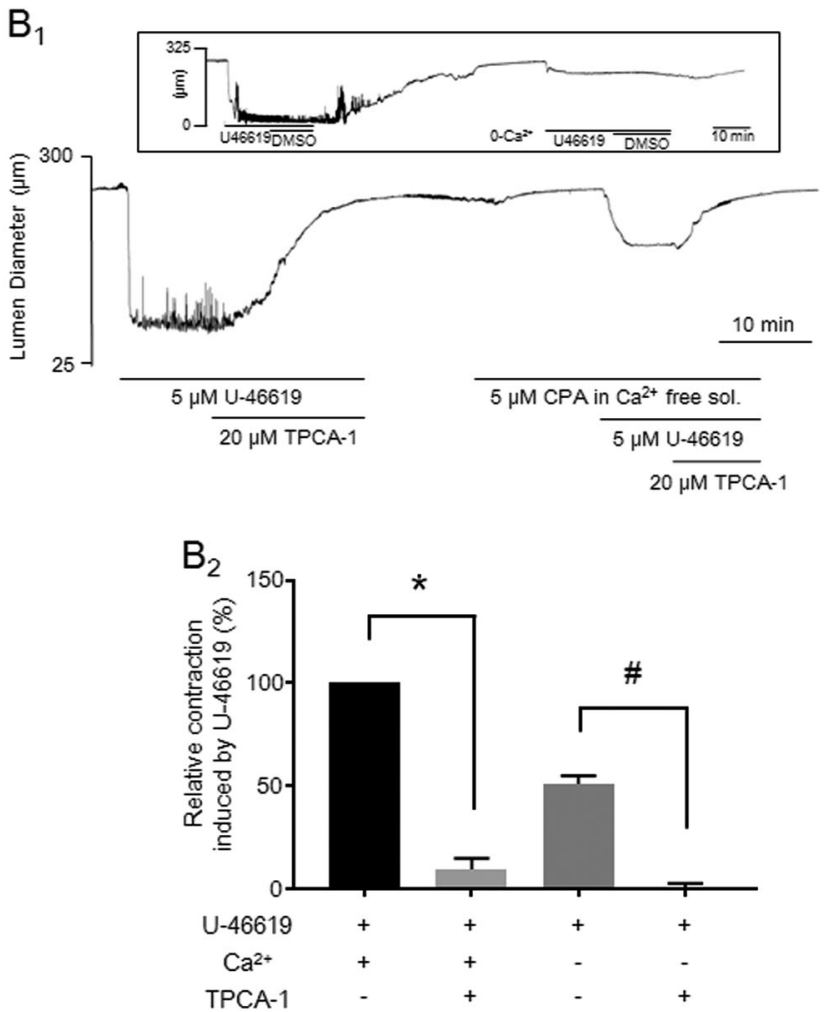

normal and $0-\mathrm{Ca}^{2+} \mathrm{KH}$ condition. $\mathbf{b}_{2}$ Percent relative contraction induced by U-46619 in mesenteric arteries $(n=5)$. ${ }^{*} p<0.05$ for U-46619-induced contraction vs U-46619-induced contraction with TPCA-1 treatment, ${ }^{*} p<0.05$ for U-46619-induced contraction in $0-\mathrm{Ca}^{2+}$ condition vs U-46619-induced contraction with TPCA-1 treatment in $0-\mathrm{Ca}^{2+}$ condition

(p-MYPT1, thr853) were reduced by co-treatment of U46619 and SC-514 compared with treatment of U-46619 (Fig. 6). In contrast, phosphorylation levels of CPI17 (thr38) and MYPT1 (thr696) were not changed by treatment of SC-514. To confirm the immunofluorescence staining results, we performed western blot analysis for phosphorylation level of MLC. In accordance with the immunofluorescence data, phosphorylation level MLC was significantly increased in the mesenteric arteries treated with U-46619. Interestingly, treatment of SC-514 significantly reduced MLC phosphorylation level (Fig. $6 \mathrm{e}_{1}, \mathrm{e}_{2}$ ).

\section{Discussion}

The major finding of this study is that IKK2 is involved in the vascular smooth muscle contractility through inhibition of MLCP activity. The SC-514, an IKK2 inhibitor, decreased $70 \mathrm{mM} \mathrm{K}^{+}$-, PE- and U-46619-induced contraction (Figs. 1, 2). When ML-7 was pre-treated, U-46619induced contraction was reduced by treatment of SC-514 (Fig. 3). Moreover, U-46619-induced vasoconstriction was 
Fig. 6 Effect of SC-514 on the phosphorylation levels of MLC, MYPT1, and CPI-17 in mesenteric arteries. a-d Immunofluorescence of phosphorylated MLC, MYPT1, and CPI-17 in mesenteric arteries. $\mathbf{a}_{1}$ Sections of resting state, resting state treated with SC-514, treatment of U-46619 and co-treatment of U-46619 and SC-514 stained for p-MLC (ser19, red) and t-MLC (green). $\mathbf{a}_{2}$ Mean data for $\mathrm{p}-\mathrm{MLC} / \mathrm{t}-\mathrm{MLC}$ expression $(n=5)$. $\mathbf{b}_{1}$ Sections of resting state, resting state treated with SC-514, treatment of U-46619 and co-treatment of U-46619 and SC-514 stained for p-MYPT1 (thr853, red) and t-MYPT1 (green). $\mathbf{b}_{2}$ Mean data for p-MYPT1 (thr853)/t-

MYPT1 expression $(n=4)$. $\mathbf{c}_{1}$ Sections of resting state, resting state treated with SC-514, treatment of U-46619 and cotreatment of U-46619 and SC514 stained for p-MYPT1 (thr696, red) and t-MYPT1 (green). $\mathbf{c}_{2}$ Mean data for $\mathrm{p}$ MYPT1 (thr696)/t-MYPT1 expression $(n=4)$. $\mathbf{d}_{1}$ Sections of resting state, resting state treated with SC-514, treatment of U-46619 and co-treatment of U-46619 and SC-514 stained for p-CPI17 (thr38, red) and smooth muscle actin (SMA, green). $\mathbf{d}_{2}$ Mean data for p-CPI17/SMA expression $(n=4)$. DAPI (blue) was used for nuclear staining. $\mathrm{e}$ Western blot analysis for MLC. $\mathbf{e}_{1}$ Representative western blot data. $\mathbf{e}_{2}$ Quantitative data for t-MLC and p-MLC $(n=3)$. $* p<0.05$ for resting state vs U-46619-induced contraction, ${ }^{\#} p<0.05$ for U-46619-induced contraction vs U-46619-induced contraction with SC-514
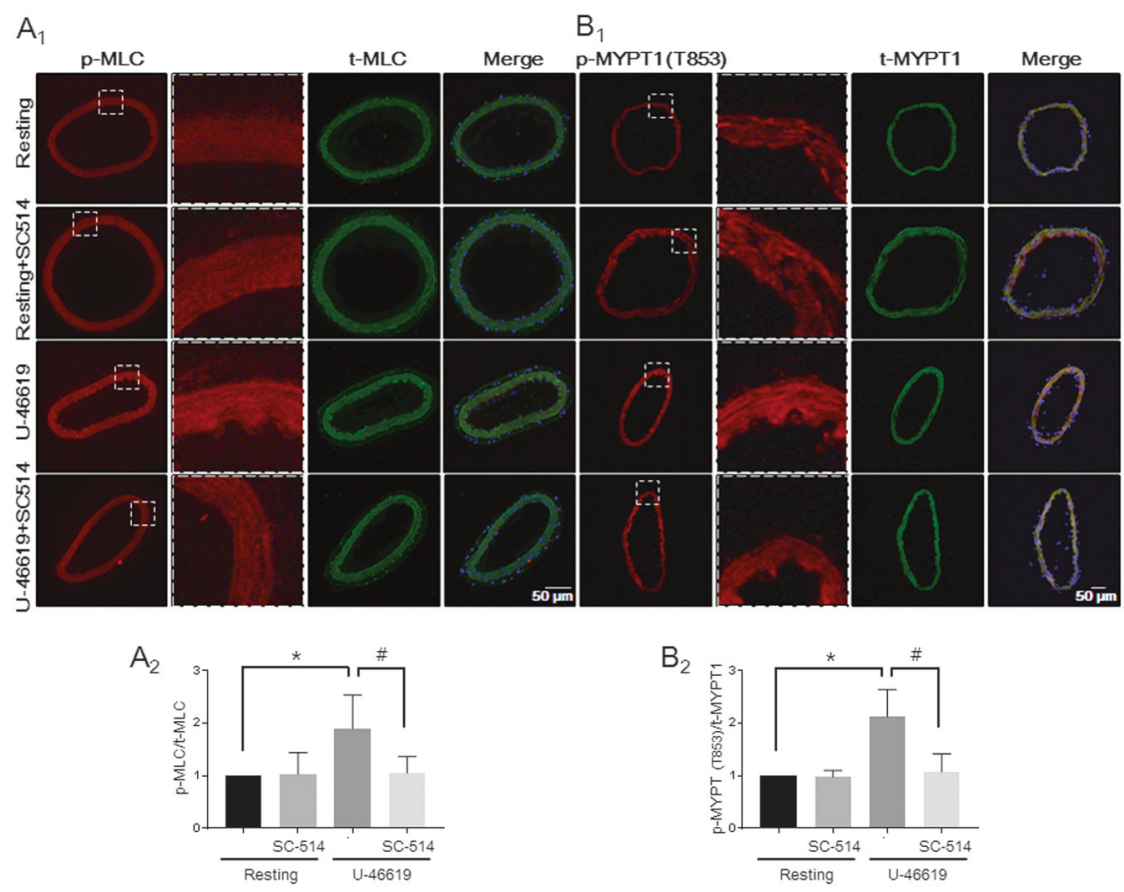

$\mathrm{B}_{2}$

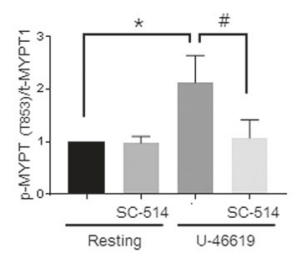

$\mathrm{C}_{1}$
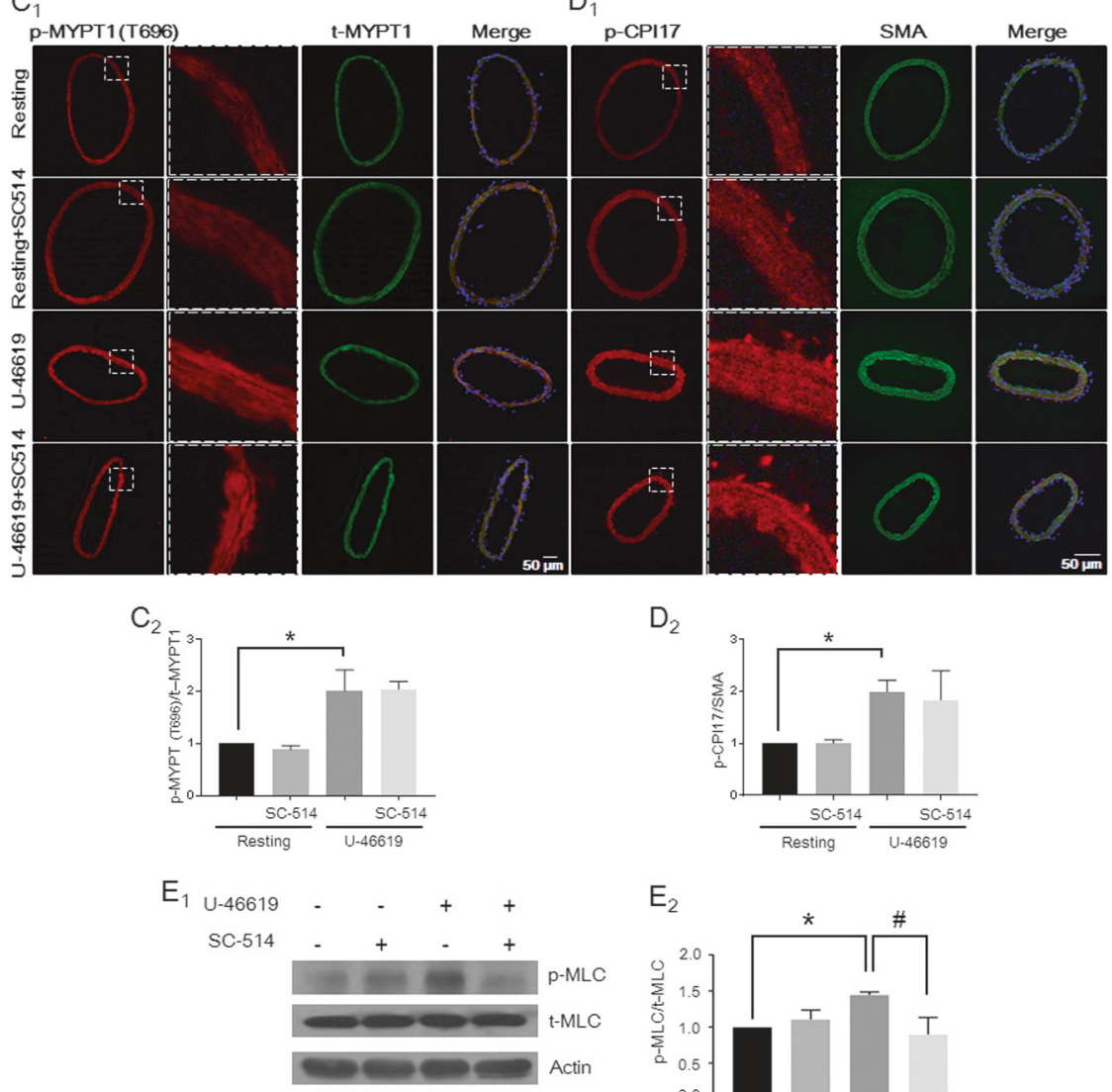

$\mathrm{D}_{2}$
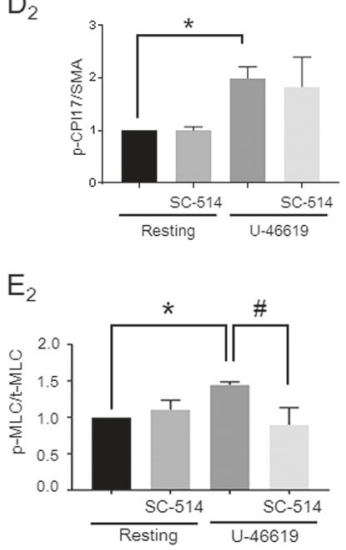

relaxed by treatment of SC-514 in the $0-\mathrm{Ca}^{2+} \mathrm{KH}$ solution (Fig. 4). Similar results were obtained in the mesenteric arteries treated with another inhibitor of IKK2, TPCA-1
(Fig. 5). We also revealed that SC-514 decreases phosphorylation level of MYPT1 at thr853 (Fig. 6). Our results implicate that IKK2 has an important role in the regulation 
of arterial tone through regulating MYPT1 activity in mesenteric arteries.

Recently, several studies reported that IKK2 acts as another MLCK and contributes to vessel contraction in rat and mouse aorta [26, 27]. In addition, it has been also reported that tumor necrosis factor- $\alpha$, the well-known regulator of IKK2, is involved in MLC phosphorylation in human vascular smooth muscle cells [27]. In the present study, we focused on investigating another role of IKK2 in MLCP regulation.

To investigate whether IKK2 acts as another MLCK or regulates the activity of MLCP, we compared the effect of SC-514 on high $\mathrm{K}^{+}$and U-46619-induced contractions. High $\mathrm{K}^{+}$-induced contraction is mainly caused by an increase in intracellular $\mathrm{Ca}^{2+}$ level [30]. On the other hand, agonists such as angiotensin, epinephrine, endothelin-1 (ET1), and thromboxane A2 (TXA2) induce contraction through two parallel signaling pathways: an increase of cytosolic $\mathrm{Ca}^{2+}$ level and activation of the RhoA/Rho-associated kinase (ROCK) pathway, which is known as $\mathrm{Ca}^{2+}$ sensitization [31, 32]. In the present study, we found that relaxation induced by SC-514 was significantly increased in the contractile response to U-46619 compared with the contractile response to high $\mathrm{K}^{+}$(Fig. 2). These data indicate that IKK2 is more involved in U-46619-induced contraction, which is associated with $\mathrm{Ca}^{2+}$-dependent and $\mathrm{Ca}^{2+}$-sensitization mechanism. Therefore, we considered that IKK2 might regulate the activity of MLCP. To further identify this hypothesis, we performed the study on the $\mathrm{Ca}^{2+}$-independent contraction. We used ML-7, a selective MLCK inhibitor, which inhibits MLCK via acting on the adenosine triphosphate (ATP)-binding site of MLCK [33]. The concentration of ML-7 used in the present study was determined with reference to previous studies done by other research groups [34, 35]. The SC-514 inhibited U-46619-induced contraction when classic MLCK was inhibited by ML-7 (Fig. 3). In addition, we confirmed that $\mathrm{Ca}^{2+}$-independent, U-46619-induced contraction was inhibited by SC-514 and TPCA-1 (Figs. 4 and 5). To strengthen these data, we measured the phosphorylation level of MLC using immunofluorescence staining and western blot analysis. Phosphorylated MLC (at ser19) was increased in U-46619induced contraction and significantly decreased by SC-514 (Fig. $6 a_{1}, e_{1}$ ). Furthermore, we also found that p-MYPT1 (thr853) was increased in U-46619-induced contraction and significantly decreased by SC-514 in the immunofluorescence staining data. However, levels of p-MYPT1 (thr696) and p-CPI17 (thr38) were not altered by treatment of SC-514 (Fig. 6). Our study revealed that IKK2 regulates the activity of MLCP through phosphorylation of MYPT1 at thr853 in mesenteric arteries.

We identified IKK2 is involved in the $\mathrm{Ca}^{2+}$ sensitization mechanism of vascular smooth muscle contraction.
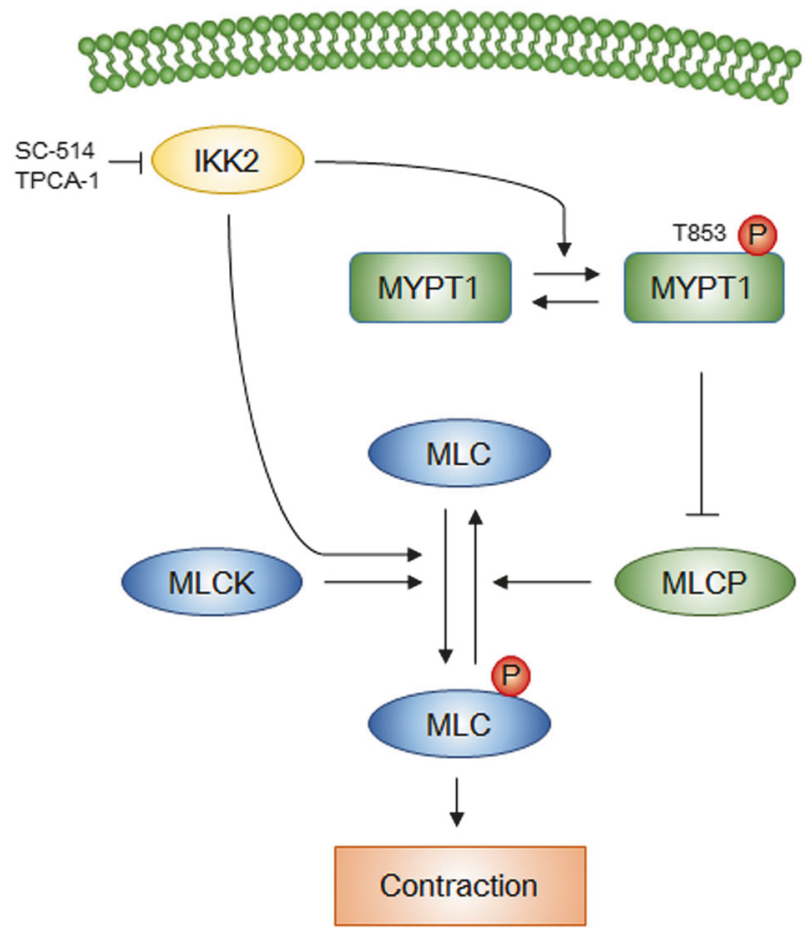

Fig. 7 Involvement of IKK2 in the MLC phosphorylation. IKK2 inhibits MLCP through phosphorylation of MYPT1 at thr853, which results in increase of MLC phosphorylation

However, there is no relationship with CPI-17 phosphorylation. Our results suggested that IKK2 regulates activity of MLCP through MYPT1 phosphorylation.

In this study, we propose another role of IKK2 in rat mesenteric arteries. Our results indicate that IKK2 involves smooth muscle contraction through regulating the activity of MLCP in rat mesenteric arteries. It has been well known that MYPT1 contributes to vascular contractile response and changes blood pressure in vivo [36]. Thus, we suggest IKK2 could be a new pharmacological target for specific therapies of various vascular diseases (Fig. 7).

\section{Disclaimer}

The funders had no role in study design, data collection and analysis, decision to publish, or preparation of the manuscript.

Acknowledgements This work was supported by the Basic Science Research Program of the National Research Foundation of Korea (NRF) funded by the Ministry of Education, Science and Technology (NRF-2016R1C1B1006504).

\section{Compliance with ethical standards}

Conflict of interest The authors declare that they have no conflict of interest. 


\section{References}

1. Jacob M, Chappell D, Becker BF. Regulation of blood flow and volume exchange across the microcirculation. Crit Care. 2016;20:319.

2. Christensen KL, Mulvany MJ. Location of resistance arteries. J Vasc Res. 2001;38:1-12.

3. Intengan HD, Schiffrin EL. Structure and mechanical properties of resistance arteries in hypertension: role of adhesion molecules and extracellular matrix determinants. Hypertension. 2000;36:312-8.

4. Kamm KE, Stull JT. The function of myosin and myosin light chain kinase phosphorylation in smooth muscle. Annu Rev Pharmacol Toxicol. 1985;25:593-620.

5. Webb RC. Smooth muscle contraction and relaxation. Adv Physiol Educ. 2003;27:201-6.

6. Mizuno Y, Isotani E, Huang J, et al. Myosin light chain kinase activation and calcium sensitization in smooth muscle in vivo. Am J Physiol Cell Physiol. 2008;295:C358-64.

7. Cho YE, Ahn DS, Morgan KG, et al. Enhanced contractility and myosin phosphorylation induced by $\mathrm{Ca}^{2+}$ )-independent MLCK activity in hypertensive rats. Cardiovasc Res. 2011;91:162-70.

8. Kitazawa T, Kitazawa K. Size-dependent heterogeneity of contractile $\mathrm{Ca}^{2+}$ sensitization in rat arterial smooth muscle. J Physiol. 2012;590:5401-23.

9. Uehata M, Ishizaki T, Satoh $\mathrm{H}$, et al. Calcium sensitization of smooth muscle mediated by a Rho-associated protein kinase in hypertension. Nature. 1997;389:990-4.

10. Chiba Y, Ueno A, Shinozaki K, et al. Involvement of RhoAmediated $\mathrm{Ca}^{2+}$ sensitization in antigen-induced bronchial smooth muscle hyperresponsiveness in mice. Respir Res. 2005;6:4.

11. Nobe K, Hashimoto T, Honda K. Two distinct dysfunctions in diabetic mouse mesenteric artery contraction are caused by changes in the Rho A-Rho kinase signaling pathway. Eur J Pharmacol. 2012;683:217-25.

12. Dimopoulos GJ, Semba S, Kitazawa K, et al. $\mathrm{Ca}^{2+}$-dependent rapid $\mathrm{Ca}^{2+}$ sensitization of contraction in arterial smooth muscle. Circ Res. 2007;100:121-9.

13. Kitazawa T, Eto M, Woodsome TP, et al. Phosphorylation of the myosin phosphatase targeting subunit and CPI-17 during $\mathrm{Ca}^{2+}$ sensitization in rabbit smooth muscle. $\mathrm{J}$ Physiol. 2003;546:879-89.

14. Somlyo AP, Somlyo AV. $\mathrm{Ca}^{2+}$ sensitivity of smooth muscle and nonmuscle myosin II: modulated by $\mathrm{G}$ proteins, kinases, and myosin phosphatase. Physiol Rev. 2003;83:1325-58.

15. Sward K, Mita M, Wilson DP, et al. The role of RhoA and Rhoassociated kinase in vascular smooth muscle contraction. Curr Hypertens Rep. 2003;5:66-72.

16. Schmid JA, Birbach A. IkappaB kinase beta (IKKbeta/IKK2/ IKBKB)--a key molecule in signaling to the transcription factor NF-kappaB. Cytokine Growth Factor Rev. 2008;19:157-65.

17. Tak PP, Firestein GS. NF-kappaB: a key role in inflammatory diseases. J Clin Invest. 2001;107:7-11.

18. Lawrence T. The nuclear factor NF-kappaB pathway in inflammation. Cold Spring Harb Perspect Biol. 2009;1:a001651.

19. Israel $\mathrm{A}$. The IKK complex, a central regulator of NF-kappaB activation. Cold Spring Harb Perspect Biol. 2010;2:a000158.

20. Tilstra JS, Gaddy DF, Zhao J, et al. Pharmacologic IKK/NFkappaB inhibition causes antigen presenting cells to undergo TNFalpha dependent ROS-mediated programmed cell death. Sci Rep. 2014;4:3631.
21. Lee JY, Kim H, Cha MY, et al. Clostridium difficile toxin A promotes dendritic cell maturation and chemokine CXCL2 expression through $\mathrm{p} 38$, IKK, and the NF-kappaB signaling pathway. J Mol Med. 87:169-80.

22. Schmidt-Supprian M, Tian J, Grant EP, et al. Differential dependence of $\mathrm{CD}^{+}{ }^{+} \mathrm{CD} 25^{+}$regulatory and natural killer-like $\mathrm{T}$ cells on signals leading to NF-kappaB activation. Proc Natl Acad Sci USA. 2004;101:4566-71.

23. Sun L, Deng L, Ea CK, et al. The TRAF6 ubiquitin ligase and TAK1 kinase mediate IKK activation by BCL10 and MALT1 in T lymphocytes. Mol Cell. 2004;14:289-301.

24. Catley MC, Sukkar MB, Chung KF, et al. Validation of the antiinflammatory properties of small-molecule IkappaB kinase (IKK)2 inhibitors by comparison with adenoviral-mediated delivery of dominant-negative IKK1 and IKK2 in human airways smooth muscle. Mol Pharmacol. 2006;70:697-705.

25. Birrell MA, Hardaker E, Wong S, et al. Ikappa-B kinase-2 inhibitor blocks inflammation in human airway smooth muscle and a rat model of asthma. Am J Respir Crit Care Med. 2005;172:962-71.

26. Ying Z, do Carmo JM, Xiang L, et al. Inhibitor kappaB kinase 2 is a myosin light chain kinase in vascular smooth muscle. Circ Res. 2013;113:562-70.

27. Chen M, Ma L, Hall JE, et al. Dual regulation of tumor necrosis factor-alpha on myosin light chain phosphorylation in vascular smooth muscle. Am J Physiol Heart Circ Physiol. 2015;308: H398-406.

28. Choi SK, Lim M, Byeon SH, et al. Inhibition of endoplasmic reticulum stress improves coronary artery function in the spontaneously hypertensive rats. Sci Rep. 2016;6:31925.

29. Choi SK, Ahn DS, Lee YH. Comparison of contractile mechanisms of sphingosylphosphorylcholine and sphingosine-1phosphate in rabbit coronary artery. Cardiovasc Res. 2009;82:324-32.

30. Kirschstein $\mathrm{T}$, Rehberg M, Bajorat $\mathrm{R}$, et al. High $\mathrm{K}^{+}$-induced contraction requires depolarization-induced $\mathrm{Ca}^{2+}$ release from internal stores in rat gut smooth muscle. Acta Pharmacol Sin. 2009;30:1123-31.

31. Somlyo AP, Somlyo AV. Signal transduction and regulation in smooth muscle. Nature. 1994;372:231-6.

32. Artamonov MV, Momotani K, Stevenson A, et al. Agonistinduced $\mathrm{Ca}^{2+}$ sensitization in smooth muscle: redundancy of Rho guanine nucleotide exchange factors (RhoGEFs) and response kinetics, a caged compound study. J Biol Chem. 2013;288:34030-40.

33. Cheng X, Wang X, Wan Y, et al. Myosin light chain kinase inhibitor ML7 improves vascular endothelial dysfunction via tight junction regulation in a rabbit model of atherosclerosis. Mol Med Rep. 2015;12:4109-16.

34. Ito K, Matsuzaki M, Sasahara T, et al. Orthovanadate-induced vasoconstriction of rat mesenteric arteries is mediated by Rho kinase-dependent inhibition of myosin light chain phosphatase. Biol Pharm Bull. 2015;38:1809-16.

35. Yang E, Cho JY, Sohn UD, et al. Calcium sensitization induced by sodium fluoride in permeabilized rat mesenteric arteries. Korean J Physiol Pharmacol. 2010;14:51-7.

36. Qiao YN, He WQ, Chen CP, et al. Myosin phosphatase target subunit 1 (MYPT1) regulates the contraction and relaxation of vascular smooth muscle and maintains blood pressure. J Biol Chem. 2014;289:22512-23. 